\title{
State Feedback Controller Design of an Active Suspension System for Vehicles Using Pole Placement Technique
}

\author{
A. Wéber, M. Kuczmann \\ Széchenyi István University, Department of Automation \\ Egyetem tér 1., 9026, Győr \\ E-mail: mandycandy.art@gmail.com
}

Abstract: The paper presents a method for designing a state feedback controller of an active suspension system of a quarter car model. This is a survey based on a specific example. The designed controller of the active suspension system improves the driving control, safety and stability, because during the ride, the periodic swinging motion generated by the road irregularities on wheels can be decreased. This periodic motion damages the driving comfort, and may cause traffic accidents. The state feedback controller is designed to stand road induced displacements. Computer simulations of the designed controller have been performed in the frame of Scilab and XCos.

Keywords: $\quad$ state feedback, pole placement, active suspension system

\section{Introduction}

Many researches performed on active suspension system have been presented in the recent years leading to more sophisticated regulatory approaches such as linear (fuzzy [1] PID controller [2]) and nonlinear control systems (artifical neural network controllers [3]). The active suspension system is a mechatronic suspension [4] and is important for improving the ride comfort. Because of the adverse impacts caused by road imbalances, the wheel can lose contact with the road, it can not deliver force, 
and therefore the driving of the vehicle becomes uncertain. The periodic swinging motion can damage the driving comfort, the car parts, the cargo, and this motion can generate health damage, too. The primary purpose of the active suspension system is to minimize the vertical displacement of the vehicle and guarantee road maintenance. For modeling and simulation, a quarter car model has been chosen (see Fig. 1).

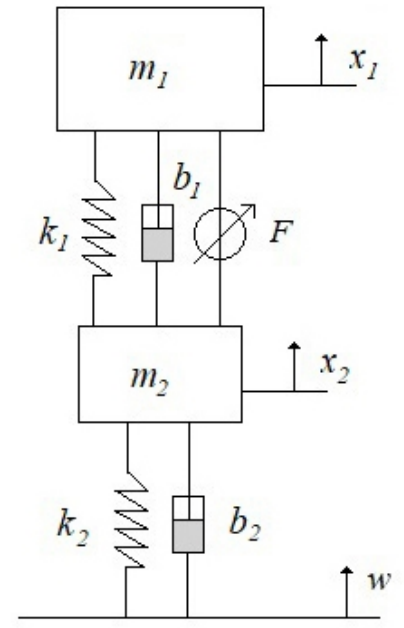

Figure 1. Quarter car model

\section{Mathematical modeling}

\subsection{The quarter car model}

Dynamic systems are described by several scientific and engineering branches and are modeled by state equations. Using differential equations, the operation of complicated dynamic systems can be modeled with relatively high precision. For defining the state 
variables of a quarter car model the Euler-Lagrange equation is used [5]:

$$
\frac{d}{d t} \frac{\partial K}{\partial \dot{x}}-\frac{\partial K}{\partial x}+\frac{\partial P}{\partial x}+\frac{\partial R}{\partial \dot{x}}=F .
$$

The equation is described by the kinetic energy $K$, the potential energy $P$ and the Rayleigh distribution $R$, as follows [6]:

$$
\begin{gathered}
K=\frac{1}{2} m_{1} \dot{x}_{1}^{2}+\frac{1}{2} m_{2} \dot{x}_{2}^{2}, \\
P=\frac{1}{2} k_{1}\left(x_{1}-x_{2}\right)^{2}+m_{1} g x_{1}+\frac{1}{2} k_{2}\left(x_{2}-w\right)^{2}+m_{2} g x_{2}, \\
R=\frac{1}{2} b_{1}\left(\dot{x}_{1}-\dot{x}_{2}\right)^{2}+\frac{1}{2} b_{2}\left(\dot{x}_{2}-\dot{w}\right)^{2},
\end{gathered}
$$

where

$m_{1}$ - sprung mass;

$m_{2}$ - un-srpung mass;

$k_{1}$ - suspension stiffness;

$k_{2}$ - tire stiffness;

$b_{1}, b_{2}$ - damping coefficients;

$F$ - action control force;

$x_{1}$ - car body displacement;

$x_{2}$ - wheel displacement;

$w$-road induced displacement.

\subsection{State-space representation of a quarter car model}

After obtaining the partial derivatives and substituting them into the Euler-Lagrange equation, the following equations are obtained:

$$
m_{1} \ddot{x}_{1}+k_{1}\left(x_{1}-x_{2}\right)+m_{1} g+b_{1}\left(\dot{x}_{1}-\dot{x}_{2}\right)=F,
$$




$$
m_{2} \ddot{x}_{2}-k_{1}\left(x_{1}-x_{2}\right)+k_{2}\left(x_{2}-w\right)+m_{2} g-b_{1}\left(\dot{x}_{1}-\dot{x}_{2}\right)+b_{2}\left(\dot{x}_{2}-\dot{w}\right)=-F .
$$

The mathematical state of a dynamic system is described by the state variables. State variables often relate to a physical process in engineering systems, where the correlation needed to store mass, pulse and current are to be calculated.

The state variables define a state-space. In this state-space, the state vector $x(t)$ is specified. The movement of the system is the displacement of its end point [7]. A state-space representation is a mathematical model of a physical system in control engineering. This is a set of input, output and state variables related by first-order differential equations.

The states of the present system are defined as follows: $\dot{x}_{1}=x_{3}, \ddot{x}_{1}=\dot{x}_{3}$. Four state variables are defined for the system:

$$
\begin{gathered}
\dot{x}_{1}=x_{3}, \\
\dot{x}_{2}=\frac{b_{1}}{m_{2}} x_{1}-\frac{b_{1}}{m_{2}} x_{2}-\frac{b_{2}}{m_{2}} x_{2}+\frac{b_{2}}{m_{2}} w+x_{4}, \\
\dot{x}_{3}=\left(\frac{b_{1}^{2}}{m_{1} m_{2}}-\frac{k_{1}}{m_{1}}\right) x_{1}-\left(\frac{b_{1}^{2}}{m_{1} m_{2}}+\frac{b_{1} b_{2}}{m_{1} m_{2}}-\frac{k_{1}}{m_{1}}\right) x_{2}-\frac{b_{1}}{m_{1}} x_{3} \\
+\frac{b_{1}}{m_{1}} x_{4}+\frac{b_{1} b_{2}}{m_{1} m_{2}} w+\frac{1}{m_{1}} f-g, \\
\dot{x}_{4}=\frac{k_{1}}{m_{2}} x_{1}-\frac{k_{1}}{m_{2}} x_{2}-\frac{k_{2}}{m_{2}} x_{2}+\frac{k_{2}}{m_{2}} w-\frac{1}{m_{2}} f-g .
\end{gathered}
$$

In the case of a linear system, the general form [8] of the state variable equations are:

$$
\dot{x}=A x+B u,
$$




$$
y=C x+D u \text {. }
$$

Here, $x$ is the state vector, $u$ and $y$ are the column vector containing exitations and responses. $A$ is the system matrix, $B, C$ and $D$ matrices contain the appropriate coefficient [9].

The state-space representation of a quarter car model is described as follow:

$$
\begin{aligned}
{\left[\begin{array}{l}
\dot{x}_{1} \\
\dot{x}_{2} \\
\dot{x}_{3} \\
\dot{x}_{4}
\end{array}\right]=} & {\left[\begin{array}{cccc}
0 & 1 & 0 \\
\frac{b_{1}}{m_{2}} & -\left(\frac{b_{1}}{m_{2}}+\frac{b_{2}}{m_{2}}\right) & 0 & 1 \\
\left(\frac{b_{1}^{2}}{m_{1} m_{2}}-\frac{k_{1}}{m_{1}}\right) & -\left(\frac{b_{1}^{2}}{m_{1} m_{2}}+\frac{b_{1} b_{2}}{m_{1} m_{2}}-\frac{k_{1}}{m_{1}}\right) & -\frac{b_{1}}{m_{1}} & \frac{b_{1}}{m_{1}} \\
\frac{k_{1}}{m_{2}} & -\left(\frac{k_{1}}{m_{2}}+\frac{k_{2}}{m_{2}}\right) & 0 & 0
\end{array}\right]\left[\begin{array}{l}
x_{1} \\
x_{2} \\
x_{3} \\
x_{4}
\end{array}\right] } \\
+ & {\left[\begin{array}{ccc}
0 & 0 & 0 \\
0 & \frac{b_{2}}{m_{2}} & 0 \\
\frac{1}{m_{1}} & \frac{b_{1} b_{2}}{m_{k_{2}}} & -g \\
-\frac{1}{m_{2}} & \frac{k_{1}}{m_{2}} & -g
\end{array}\right]\left[\begin{array}{c}
F \\
w \\
1
\end{array}\right] . }
\end{aligned}
$$

The performance parameters of the vehicle are given in Table 1. [6]. After substituting the values, the state-space representation as follow:

$$
\begin{aligned}
{\left[\begin{array}{l}
\dot{x}_{1} \\
\dot{x}_{2} \\
\dot{x}_{3} \\
\dot{x}_{4}
\end{array}\right]=} & {\left[\begin{array}{cccc}
0 & 0 & 1 & 0 \\
12.5 & -40.5 & 0 & 1 \\
-59.482759 & 11.206897 & -1.7241379 & 1.7241379 \\
587.5 & -5337.5 & 0 & 0
\end{array}\right]\left[\begin{array}{l}
x_{1} \\
x_{2} \\
x_{3} \\
x_{4}
\end{array}\right] } \\
+ & {\left[\begin{array}{ccc}
0 & 0 & 0 \\
0 & 28 & 0 \\
0.0034483 & 48.275862 & -9.81 \\
-0.025 & 4750 & -9.81
\end{array}\right]\left[\begin{array}{c}
F \\
w \\
1
\end{array}\right] . }
\end{aligned}
$$

The output variable of the quarter car model is as follows: 


$$
y=\left[\begin{array}{llll}
1 & 0 & 0 & 0
\end{array}\right]\left[\begin{array}{l}
x_{1} \\
x_{2} \\
x_{3} \\
x_{4}
\end{array}\right]+\left[\begin{array}{lll}
0 & 0 & 0
\end{array}\right]\left[\begin{array}{c}
F \\
w \\
1
\end{array}\right]
$$

Table 1. Parameters

\begin{tabular}{|c|c|c|}
\hline Parameters & Value & Unit \\
\hline$m_{1}$ & 290 & $\mathrm{~kg}$ \\
\hline$m_{2}$ & 40 & $\mathrm{~kg}$ \\
\hline$k_{1}$ & 23500 & $\mathrm{~N} / \mathrm{m}$ \\
\hline$k_{2}$ & 190000 & $\mathrm{~N} / \mathrm{m}$ \\
\hline$b_{1}$ & 500 & $\mathrm{~N} / \mathrm{m} / \mathrm{s}$ \\
\hline$b_{2}$ & 1220 & $\mathrm{~N} / \mathrm{m} / \mathrm{s}$ \\
\hline$g$ & 9.81 & $\mathrm{~m} / \mathrm{s}^{2}$ \\
\hline
\end{tabular}

\section{Simulations of the quarter car model}

\subsection{Full state feedback}

Controllability is an important property of a controlled plant. The system can be controlled when the rank of controllability matrix $M_{c}$ is maximal, i.e. the matrix is invertible if the determinant of the matrix is not zero [10]. The Kalman's controllability matrix looks as follow $(n=4)$ :

$$
\begin{gathered}
M_{c}=\left[\begin{array}{lllll}
b & A b & A^{2} b & \ldots & A^{n-1} b
\end{array}\right], \\
M_{c}=\left[\begin{array}{cccc}
0 & 0.0034483 & -0.0490488 & -0.4007186 \\
0 & -0.025 & 1.0556034 & 92.098313 \\
0.0034483 & -0.0490488 & -0.4007186 & 248.99601 \\
-0.025 & 0 & 135.46336 & -5663.0995
\end{array}\right] .
\end{gathered}
$$


After determining the controllability matrix, Ackermann's pole placement can be used because the state transformation and the feedback matrix can be directly given [6]. Because the system is controllable, Ackermann's pole placement is used for the state feedback. Ackermann's formula is a control design method for solving the pole allocation problem.

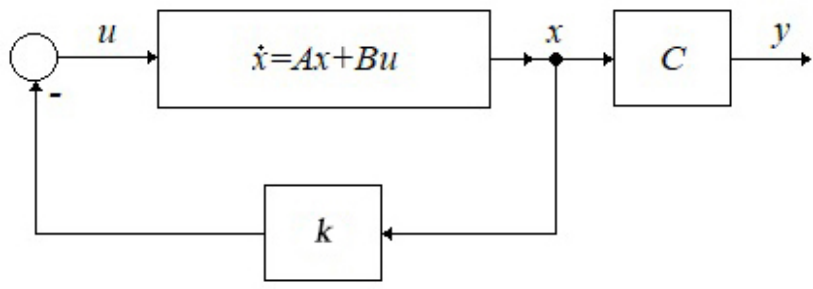

Figure 2. State feedback in continuous time

The task is to move the system's egienvalues to new places in the closed loop system. This is the pole placement, which is why the state feedback $k$ is to be determined, (see Fig. 2 [10]). The polynomial of a closed loop system in general case is

$$
\lambda^{n}+p_{1} \lambda^{n-1}+p_{2} \lambda^{n-2} \ldots+p_{n}=0 .
$$

When using the pole placement method, the eigenvalues are changed, as it can be written as:

$$
\phi_{c l}(\lambda)=\left|\lambda E-\left(A-B k^{T}\right)\right|=0 .
$$

The eigenvalues of the original system as follows:

$$
\lambda=\left[\begin{array}{cc}
-20.40805 & +70.147933 i \\
-20.40805 & -70.147933 i \\
-0.7040194 & +8.4630446 i \\
-0.7040194 & -8.4630446 i
\end{array}\right]
$$


The new poles are selected as:

$$
p=\left[\begin{array}{llll}
-200 & -30 & -30 & -30
\end{array}\right]
$$

and the gain vector has been designed by Ackerman's formula,

$$
k=\left[\begin{array}{llll}
237767.73 & -221169.83 & 32173.44 & -5473.3186
\end{array}\right] .
$$

Using the pole placement method, the new eigeinvalues of the system are as follows:

$$
\lambda=\left[\begin{array}{cc}
-200 & \\
-30.001573 & \\
-29.999214 & +0.0013619 i \\
-29.999214 & -0.0013619 i
\end{array}\right] \text {. }
$$

\subsection{Simulating the system}

To realize simulations Scilab program with XCos interface has been used. In the simulation two cases have been examined; the first when the displacement induced by the road is zero, the second when this displacement is $50 \mathrm{~mm}$. There simulations are analysed with and without the designed control.

\subsubsection{Modeling without controller}

If $w=0$, then the gravitational force is pressed for the car body (see Fig. 3) and this showed that, the system left alone is set to a stationarity state after some swing. In case of $w=50 \mathrm{~mm}$ jump, car body displacement is affected by road induced displacement (see Fig. 4), the system initially leaving it goes out of the steady state for 10 seconds when it reaches a pothole, causing mass $m_{1}$ to swing movement, 10 seconds after the transient section becomes steady state. It can be seen that this value is $50 \mathrm{~mm}$ higher.

\subsubsection{Modeling with controller}

In case when there is no road induced displacement, but there is a controller (see Fig. 5), it can be seen the swings are eliminated, the stationary state is smoother. By the 
A. Wéber and M. Kuczmann - Acta Technica Jaurinensis, Vol. 12, No. 3, pp. 178-190, 2019

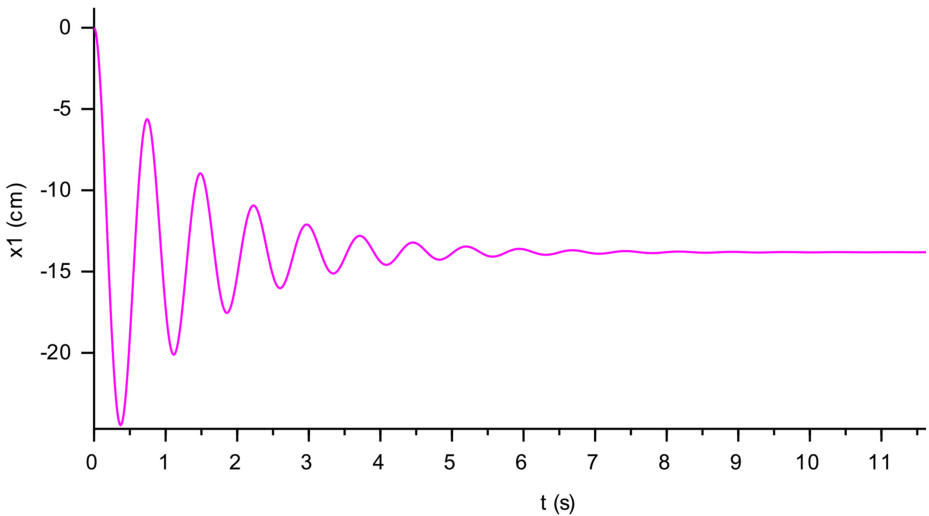

Figure 3. $w=0$ without controller

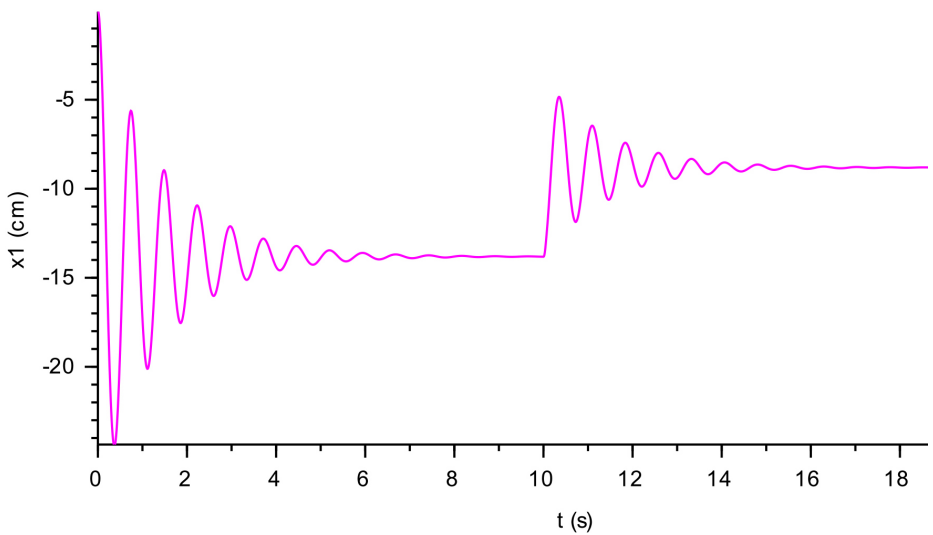

Figure 4. $w=0.05$ without controller 
reason of the design of the controller, damping force is more effective for the transient phase. Car drivers, travelers, cargoes are more favorable to this situation.

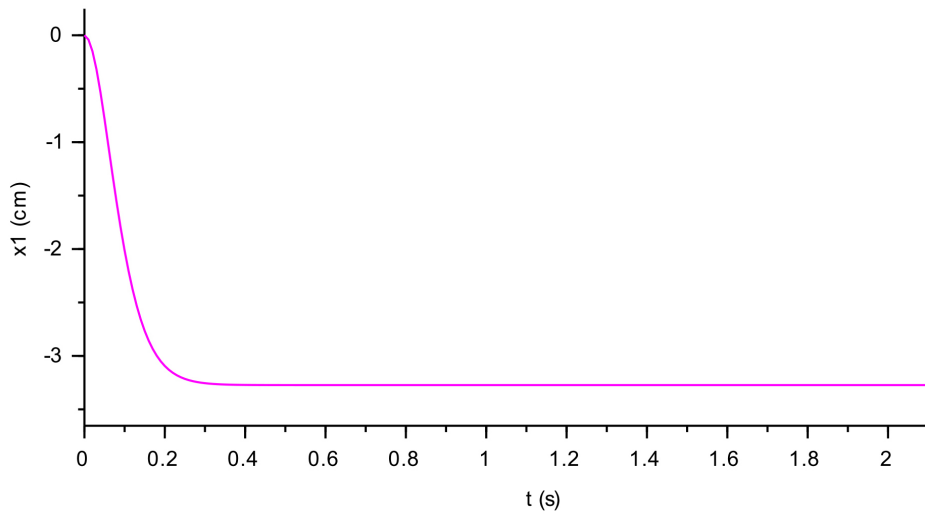

Figure 5. $w=0$ with controller

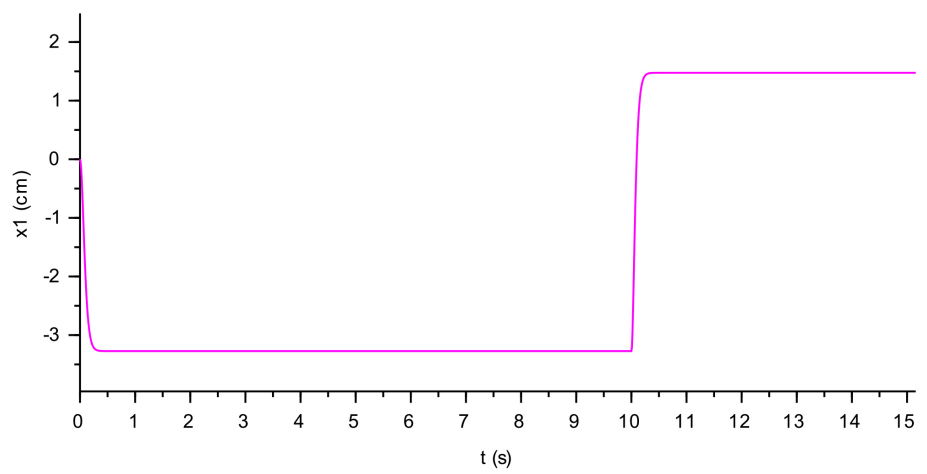

Figure 6. $w=0.05$ with controller

The effect of $50 \mathrm{~mm}$ road induced displacement is visible (see Fig. 6). Swinging 
motions disappear, 10 seconds after reaching the pothole, and after jumping the stationary state is supervened without swinging. There is state-space equation (22) without road induced displacement and state feedback (see Fig. 7).

$$
\dot{x}=A x+B_{1} u+B_{2} w+B_{3} 1
$$

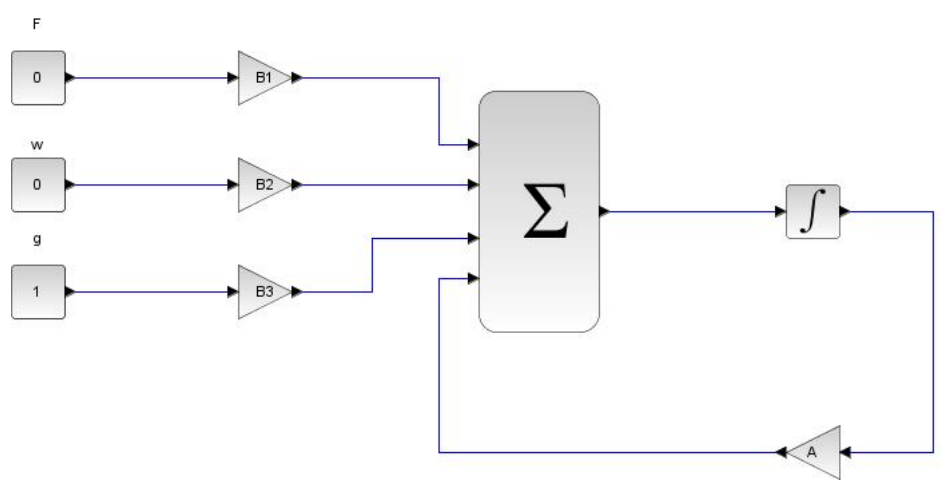

Figure 7. State-space equation model in Xcos

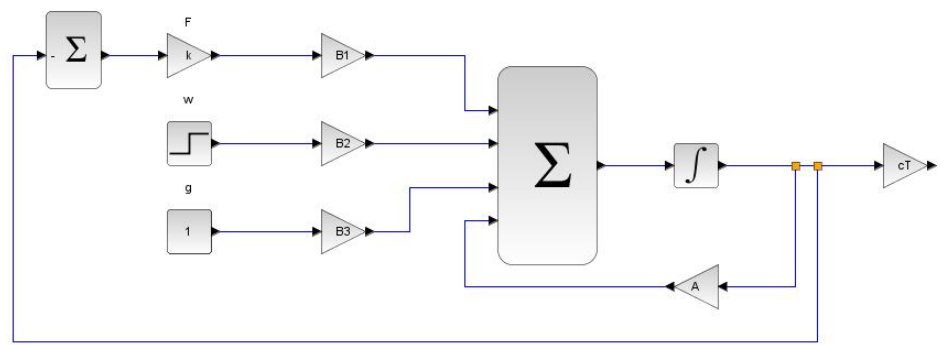

Figure 8. State feedback model in Xcos 
The state feedback model is visible, where $k$ is the gain factor (see Fig. 8) The road induced displacement and gravitational acceleration react the system. There isn't reference signal.

\section{Conclusion}

Designing of the active suspension system of a quarter car model is produced different results besides changing road induced displacement. By the simulation results, the model has much better features with the designed controller. The simulation result of the active suspension system showed that the swinging motion were gone, the stationary state quickly entered, which favored the driver, the passengers, so avoiding cargo damage.

\section{References}

[1] A. Hofmann, M. Hanss, Fuzzy arithmetical controller design for active road vehicle suspension in the presence of uncertainties, 2017 22nd International Conference on Methods and Models in Automation and Robotics (MMAR) (2017) pp. 582-587doi : 10.1109/MMAR.2017.8046893.

[2] L. Bao, S. Chen, S. Yu, Research on active fault-tolerant control on active suspension of vehicle based on fuzzy pid control, Chinese Automation Congress (CAC) (2017) pp. 5911-5916doi:10.1109/CAC.2017.8243840.

[3] V. Vidya, M. Dharmana, Model reference based intelligent control of an active suspension system for vehicles, International Conference on circuits Power and Computing Technologies [ICCPCT] (2017) pp. 1-5doi : $10.1109 /$ ICCPCT . 2017.8074362 .

[4] L. R. Miller, Tuning passive, semi-active, and fully active suspension systems, Proceedings of the 27th IEEE Conference on Decision and Control (1988) pp. 2047-2053doi:10.1109/CDC.1988.194694.

[5] B. Lantos, Control Systems Theory and Design II., 1st Edition, Akadémiai Kiadó, Budapest, 2003. 
[6] J. Bokor, P. Gáspár, State-space representation, in: L. Nádai (Ed.), Control Technology with Vehicle Dynamics Applications, 1st Edition, Typotex Elektronikus Kiadó Kft., Budapest, 2008, p. 125.

[7] L. Keviczky, R. Bars, H. J., A. Barta, C. Bányász, Control Engineering, 1st Edition, Universitas-Győr Kht., Győr, 2006.

[8] J. Bokor, P. Gáspár, A. Soumelidis, Control Engineering II., 1st Edition, Typotex Elektronikus Kiadó Kft., Budapest, 2011.

[9] M. Kuczmann, Signals and Systems, 1st Edition, Universitas-Győr Kht., Győr, 2005.

[10] B. Lantos, Control Systems Theory and Design I., 1st Edition, Akadémiai Kiadó, Budapest, 2000. 